Volume 32 (2022) 157-175

DOI: $10.24330 /$ ieja.1077596

\title{
CATEGORY OF $n$-FCP-GR-PROJECTIVE MODULES WITH RESPECT TO SPECIAL COPRESENTED GRADED MODULES
}

\author{
Mostafa Amini, Driss Bennis and Soumia Mamdouhi \\ Received: 13 August 2021; Revised: 19 October 2021; Accepted: 8 November 2021 \\ Communicated by Tuğçe Pekacar Çalcı
}

\begin{abstract}
Let $R$ be a ring graded by a group $G$ and $n \geq 1$ an integer. We introduce the notion of $n$-FCP-gr-projective $R$-modules and by using of special finitely copresented graded modules, we investigate that (1) there exist some equivalent characterizations of $n$-FCP-gr-projective modules and graded right modules of $n$-FCP-gr-projective dimension at most $k$ over $n$-gr-cocoherent rings, (2) $R$ is right $n$-gr-cocoherent if and only if for every short exact sequence $0 \rightarrow A \rightarrow B \rightarrow C \rightarrow 0$ of graded right $R$-modules, where $B$ and $C$ are $n$-FCP-gr-projective, it follows that $A$ is $n$-FCP-gr-projective if and only if ( $g r$ $\left.\mathcal{F} \mathcal{C P}{ }_{n}, g r-\mathcal{F C P}{ }_{n}^{\perp}\right)$ is a hereditary cotorsion theory, where $g r-\mathcal{F C P}{ }_{n}$ denotes the class of $n$-FCP-gr-projective right modules. Then we examine some of the conditions equivalent to that each right $R$-module is $n$-FCP-gr-projective.
\end{abstract}

Mathematics Subject Classification (2020): 16D80, 16E05, 16E30, 16E65 16P70

Keywords: $n$-gr-Cocoherent ring, special gr-copresented module, $n$-FCP-grprojective module

\section{Introduction}

In 1969, Jans in [11] gave a definition of finitely cogenerated modules as a dual notion of finitely generated modules when he introduced co-Noetherian rings as a dual notion of Noetherian rings. A right $R$-module $M$ is said to be finitely cogenerated if for every family $\left\{M_{i}\right\}_{i \in I}$ of submodules of $M$ with $\cap_{i \in I} M_{i}=0$, there is a finite subset $J \subset I$ such that $\cap_{i \in J} M_{i}=0$. In 1994, Costa in [7] introduced the notion of $n$-coherent rings for a nonnegative integer $n$. A left $R$-module $M$ is said to be $n$-presented if it has a finite $n$-presentation, that is, there exists an exact sequence $F_{n} \rightarrow F_{n-1} \rightarrow \cdots \rightarrow F_{1} \rightarrow F_{0} \rightarrow M \rightarrow 0$ with each $F_{i}$ finitely generated free $R$-module, and a ring $R$ is called left $n$-coherent if every $n$-presented left $R$-module is $(n+1)$-presented, for more details see [6,8].

As we know, cocoherent rings as a dual notion of coherent rings have been characterized in various ways, and many nice properties were obtained for such rings in $[12,19,22]$. In 1999, Weimin Xue in [24] via finitely cogenerated modules 
introduced $n$-copresented modules and $n$-cocoherent rings as a dual notion of $n$ presented modules and $n$-coherent rings, respectively. A right $R$-module $M$ is said to be $n$-copresented if there is an exact sequence $0 \rightarrow M \rightarrow E^{0} \rightarrow E^{1} \rightarrow \cdots \rightarrow E^{n}$ of right $R$-modules, where each $E^{i}$ is finitely cogenerated injective. A ring $R$ is called right $n$-cocoherent if every $n$-copresented $R$-module is $(n+1)$-copresented. $n$-cocoherent rings have been studied by several authors (see, for example $[1,2,5,21]$ ).

The homological theory of graded rings and modules is a classical topic in algebra, because of its applications in algebraic geometry, see $([14,15,16])$. Several authors have investegated the graded aspect of some notions in relative homological algerbra. For example, Asensio et al. in [4] introduced the notions of FP-gr-injective modules, then Yang and Liu in [18] investigated homological behavior of the FPgr-injective modules on gr-coherent rings. Recently in 2018, Zhao, Gao and Huang [20] gave a definition of $n$-presented graded modules and $n$-gr-coherent rings and also, by using of $n$-presented graded modules, they introduced the concept of $n$-FPgr-injective and $n$-gr-flat modules, and then examined the homological behavior of these modules over $n$-gr-coherent rings. In case $n=1$, see $[13,18]$.

The aim of this paper is to introduce and study $n$-copresented graded right modules, $n$-gr-cocoherent right rings and $n$-FCP-gr-projective right modules as a dual notion of $n$-presented graded left modules, $n$-gr-coherent left rings and $n$-FP-grinjective left modules, respectively. Then, we study the relative homological theory of these modules and also, the properties of special finitely copresented graded modules, defined via finitely cogenerated gr-injective resolutions of $n$-copresented graded modules, play a crucial role.

This paper is organized in three sections as follows:

In Section 2, some fundamental concepts and some preliminary results are stated.

In Section 3 , we first introduce the notions of $n$-copresented graded right modules, special gr-cogenerated and special gr-copresented modules with respect to any $n$-copresented graded right module, and also $n$-cocoherent graded right rings (or, right $n$-gr-cocoherent rings). Then via $n$-copresented graded modules, we give a concept of $n$-FCP-gr-projective right modules and investigate some characterizations of these modules. In this section, examples are given in order to show that $m$-FCP-gr-projectivity does not imply $n$-FCP-gr-projectivity for any $m>n$.

In Section 4, we prove that there exist some equivalent characterizations of graded right modules of $n$-FCP-gr-projective dimension at most $k$ on right $n$-grcoherent rings. We obtain some equivalent characterizations of right $n$-gr-cocoherent rings in terms of $n$-FCP-gr-projective right modules on the short exact sequences. For example, $R$ is a right $n$-gr-cocoherent ring if and only if for every exact sequence 
$0 \rightarrow A \rightarrow B \rightarrow C \rightarrow 0$ of graded right $R$-modules, where $B$ and $C$ are $n$-FCPgr-projective, it follows that $A$ is $n$-FCP-gr-projective if and only if $\left(g r-\mathcal{F C P}{ }_{n}\right.$, $\left.g r-\mathcal{F C P}{ }_{n}^{\perp}\right)$ is hereditary cotorsion theory, where $g r-\mathcal{F C P}{ }_{n}$ denotes the class of $n$ FCP-gr-projective right modules. Moreover, if gr- $\mathcal{F} \mathcal{I}_{n}$ is a class of $n$-FP-gr-injective left modules, then $\left.(g r-\mathcal{F C P})_{n}\right)^{*} \subseteq g r-\mathcal{F} \mathcal{I}_{n}$. Hence, we prove that every graded right $R$-module is $n$-FCP-gr-projective if and only if $\left(g r-\mathcal{F C P}{ }_{n}, g r-\mathcal{F C P}{ }_{n}^{\perp}\right)$ is a perfect hereditary cotorsion theory and $N \in g r-\mathcal{F C} \mathcal{P}_{n}^{\perp}$ has an $n$-FCP-gr-projective cover with the unique mapping property if and only if $R$ is right $n$-gr-cocoherent and $N$ is $n$-FCP-gr-projective if and only if $N(\sigma)$ is gr-injective for any $\sigma \in G$.

\section{Preliminaries}

Throughout this paper, all rings considered are associative with identity element and the $R$-modules are unital. By Mod- $R$ and $R$-Mod we will denote the Grothendieck category of all right $R$-modules and left $R$-modules, respectively.

Let $G$ be a multiplicative group with neutral element $e$. A graded ring $R$ is a ring with identity 1 together with a direct decomposition $R=\bigoplus_{\sigma \in G} R_{\sigma}$ (as additive subgroups) such that $R_{\sigma} R_{\tau} \subseteq R_{\sigma \tau}$ for all $\sigma, \tau \in G$. Thus, $R e$ is a subring of $R$, $1 \in R e$ and $R_{\sigma}$ is an $R e$-bimodule for every $\sigma \in G$. A graded right (resp. left) $R$-module is a right (resp. left) $R$-module $M$ endowed with an internal direct sum decomposition $M=\bigoplus_{\sigma \in G} M_{\sigma}$, where each $M_{\sigma}$ is a subgroup of the additive group of $M$ such that $M_{\tau} R_{\sigma} \subseteq M_{\tau \sigma}$ for all $\sigma, \tau \in G$. For any graded right $R$-modules $M$ and $N$, set $\operatorname{Hom}_{\mathrm{gr}-R}(M, N):=\left\{f: M \rightarrow N \mid f\right.$ is $R$-linear and $f\left(M_{\sigma}\right) \subseteq N_{\sigma}$ for any $\sigma \in G\}$, which is the group of all morphisms from $M$ to $N$ in the category gr- $R$ of all graded right $R$-modules ( $R$-gr will denote the category of all graded left $R$ modules). It is well known that gr- $R$ is a Grothendieck category. An $R$-linear map $f: M \rightarrow N$ is said to be a graded morphism of degree $\tau$ with $\tau \in G$ if $f\left(M_{\sigma}\right) \subseteq$ $M_{\sigma \tau}$ for all $\sigma \in G$. Graded morphisms of degree $\sigma$ build an additive subgroup $\operatorname{HOM}_{R}(M, N)_{\sigma}$ of $\operatorname{Hom}_{R}(M, N)$. Then $\operatorname{HOM}_{R}(M, N)=\bigoplus_{\sigma \in G} \operatorname{HOM}_{R}(M, N)_{\sigma}$ is a graded abelian group of type $G$. We will denote by $\operatorname{Ext}_{\mathrm{gr}-R}^{i}$ and $\mathrm{EXT}_{R}^{i}$ the right derived functors of $\mathrm{Hom}_{\mathrm{gr}-R}$ and $\mathrm{HOM}_{R}$, respectively. Given a graded right $R$ module $M$, the graded character module of $M$ is defined as $M^{*}:=\operatorname{HOM}_{\mathbb{Z}}(M, \mathbb{Q} / \mathbb{Z})$, where $\mathbb{Q}$ is the rational numbers field and $\mathbb{Z}$ is the integers ring. It is easy to see that $M^{*}=\bigoplus_{\sigma \in G} \operatorname{Hom}_{\mathbb{Z}}\left(M_{\sigma^{-1}}, \mathbb{Q} / \mathbb{Z}\right)$.

Let $M$ be a graded right $R$-module and $N$ a graded left $R$-module. The abelian group $M \bigotimes_{R} N$ may be graded by putting $\left(M \bigotimes_{R} N\right)_{\sigma}$ with $\sigma \in G$ to be the additive subgroup generated by elements $x \otimes y$ with $x \in M_{\alpha}$ and $y \in N_{\beta}$ such that $\alpha \beta=\sigma$. The object of $\mathbb{Z}$-gr thus defined will be called the graded tensor product of 
$M$ and $N$. If $M$ is a graded right $R$-module and $\sigma \in G$, then $M(\sigma)$ is the graded right $R$-module obtained by putting $M(\sigma)_{\tau}=M_{\tau \sigma}$ for any $\tau \in G$. The graded

module $M(\sigma)$ is called the $\sigma$-suspension of $M$. We may regard the $\sigma$-suspension as an isomorphism of categories $T_{\sigma}:$ gr- $R \rightarrow$ gr- $R$, given on objects as $T_{\sigma}(M)=M(\sigma)$ for any $M \in$ gr- $R$.

The forgetful functor $U: \operatorname{gr}-R \rightarrow \operatorname{Mod}-R$ associates to $M$ the underlying ungraded $R$-module. This functor has a right adjoint $F$ which associated to $M \in$ Mod- $R$ the graded $R$-module $F(M)=\bigoplus_{\sigma \in G}\left({ }^{\sigma} M\right)$, where each ${ }^{\sigma} M$ is a copy of $M$ written $\left\{{ }^{\sigma} x: x \in M\right\}$ with $R$-module structure defined by $r *^{\tau} x={ }^{\sigma \tau}(r x)$ for each $r \in R_{\sigma}$. If $f: M \rightarrow N$ is $R$-linear, then $F(f): F(M) \rightarrow F(N)$ is a graded morphism given by $F(f)\left({ }^{\sigma} x\right)={ }^{\sigma} f(x)$.

The injective (resp. projective) objects of gr- $R$ will be called injective graded right (resp. projective graded right) modules because $M$ is gr-injective (resp. grprojective) in gr- $R$ if and only if it is a injective (resp. projective) graded right module. Similarly, it is defined for the graded left modules in $R$-gr. Let $n \geq 0$ be an integer. Then, a graded left $R$-module $F$ is called $n$-presented [20] if there exists an exact sequence $P_{n} \rightarrow P_{n-1} \rightarrow \cdots \rightarrow P_{1} \rightarrow P_{0} \rightarrow F \rightarrow 0$ in $R$-gr with each $P_{i}$ is finitely generated free $R$-module. A graded ring $R$ is called left $n$-gr-coherent if each $n$-presented graded left $R$-module is $(n+1)$-presented. A graded left module $M$ is called $n$-FP-gr-injective if $\operatorname{EXT}_{R}^{n}(F, M)=0$ for any $n$-presented graded left $R$-module $F$. A graded left module $M$ is called $n$-gr-flat if $\operatorname{Tor}_{n}^{R}(M, F)=0$ for any $n$-presented graded left $R$-module $F$, see [20].

For a graded ring $R$, let $\mathcal{X}$ be a class of graded right $R$-modules and $M$ a graded left $R$-module. Following [3,20], we say that a graded morphism $f: X \rightarrow M$ is an $\mathcal{X}$-precover of $M$ if $X \in \mathcal{X}$ and $\operatorname{HOM}_{R}\left(X^{\prime}, X\right) \rightarrow \operatorname{HOM}_{R}\left(X^{\prime}, M\right) \rightarrow 0$ is exact for all $X^{\prime} \in \mathcal{X}$. Moreover, if whenever a graded morphism $g: X \rightarrow X$ such that $f g=f$ is an automorphism of $X$, then $f: X \rightarrow M$ is called an $\mathcal{X}$-cover of $M$. The class $\mathcal{X}$ is called (pre)covering if each object in $g r-R$ has an $\mathcal{X}$-(pre)cover. $\mathcal{X}$-envelope and $\mathcal{X}$-preenvelope are defined dually. Recall that a $\mathcal{X}$-cover $\phi: M \rightarrow N$ has the unique mapping property if for any homomorphism $f: A \rightarrow N$ with $A \in \mathcal{X}$, there exists a unique $g: A \rightarrow M$ such that $\phi g=f$.

\section{3. $n$-FCP-gr-projective modules}

In this section, we first introduce the special gr-copresented and special grcogenerated modules via $n$-copresented graded right modules. Then by using of these modules, some properties of $n$-FCP-gr-projective modules are discussed. 
Definition 3.1. Let $n \geq 0$ be an integer. Then, a graded right $R$-module $U$ is called $n$-copresented if there exists an exact sequence

$$
0 \longrightarrow U \longrightarrow E^{0} \longrightarrow E^{1} \longrightarrow \cdots \longrightarrow E^{n-1} \longrightarrow E^{n}
$$

in gr- $R$ with each $E^{i}$ is finitely cogenerated injective. Set $K^{n-1}=\operatorname{Coker}\left(E^{n-2} \rightarrow\right.$ $\left.E^{n-1}\right)$ and $K^{n}=\operatorname{Coker}\left(E^{n-1} \rightarrow E^{n}\right)$. Then, we shall say the sequence

$$
\Delta^{\prime}: 0 \longrightarrow K^{n-1} \longrightarrow E^{n} \longrightarrow K^{n} \longrightarrow 0
$$

in $\operatorname{gr}-R$ is a special short exact sequence. Moreover, we call the objects $K^{n}$ and $K^{n-1}$ special finitely cogenerated and special finitely copresented graded (special gr-cogenerated and special gr-copresented for short) right $R$-modules, respectively. Then, it follows that $\operatorname{EXT}_{R}^{1}\left(M, K^{n-1}\right) \cong \operatorname{EXT}_{R}^{n}(M, U)$ for any graded right $R$ module $M$. Also, a short exact sequence $0 \rightarrow A \rightarrow B \rightarrow C \rightarrow 0$ in gr- $R$ is called special gr-copure, if for every special gr-copresented $K^{n-1}$, there exists the following exact sequence:

$$
0 \longrightarrow \operatorname{HOM}_{R}\left(C, K^{n-1}\right) \longrightarrow \operatorname{HOM}_{R}\left(B, K^{n-1}\right) \longrightarrow \operatorname{HOM}_{R}\left(A, K^{n-1}\right) \longrightarrow 0,
$$

where $A$ is said to be special gr-copure in $B$. A graded ring $R$ is called right $n$-grcocoherent if each $n$-copresented graded right $R$-module is $(n+1)$-copresented.

The following lemma is the graded version of [23, Theorem 3].

Lemma 3.2. Let $0 \rightarrow A \rightarrow B \rightarrow C \rightarrow 0$ in gr-R be a short exact sequence. Then the following statements hold for any $n \geq 0$.

(1) If $A$ and $C$ are $n$-copresented, then so is $B$.

(2) If $C$ is $n$-copresented and $B$ is $(n+1)$-copresented, then $A$ is $(n+1)$ copresented.

(3) If $A$ and $B$ are $(n+1)$-copresented, then $C$ is $n$-copresented.

Definition 3.3. Let $n \geq 1$ be an integer. A graded right $R$-module $M$ is called $n$-FCP-gr- projective if $\operatorname{EXT}_{R}^{n}(M, U)=0$ for any $n$-copresented graded right $R$ module $U$.

Notice that for ungraded, a right $R$-module $M$ is called $n$-FCP-projective if $\operatorname{Ext}_{R}^{n}(M, U)=0$ for any $n$-copresented right $R$-module $U$, and in case $n=1, M$ is called FCP-projective, see [21].

Remark 3.4. (1) Every $m$-copresented graded right $R$-module is $n$-copresented for any $m \geq n$.

(2) If $n=1$, then every 1-FCP-gr- projective is FCP-gr- projective. 
(3) If $M$ is $n$-FCP-gr- projective and $U$ is $(n+1)$-copresented graded right $R$ module, then there exists an exact sequence $0 \rightarrow U \rightarrow E^{0} \rightarrow C \rightarrow 0$ in gr- $R$, where $E^{0}$ is finitely cogenerated injective and $C$ is $n$-copresented by Lemma 3.2. So we deduce that the sequence $0 \rightarrow \operatorname{EXT}_{R}^{n}(M, C) \rightarrow \mathrm{EXT}_{R}^{n+1}(M, U) \rightarrow 0$ is exact. But, $\operatorname{EXT}_{R}^{n}(M, C)=0$ since $M$ is $n$-FCP-gr-projective and $C$ is $n$-copresented. Hence, $\operatorname{EXT}_{R}^{n+1}(M, U)=0$ and consequently $M$ is $(n+1)$-FCP-gr- projective. Therefore,

$$
g r-\mathcal{F C P} \mathcal{P}_{1} \subseteq g r-\mathcal{F C P} \mathcal{P}_{2} \subseteq \cdots \subseteq g r-\mathcal{F C P}{ }_{n} \subseteq g r-\mathcal{F C P}{ }_{n+1} \subseteq \cdots .
$$

In general, $m$-FCP-gr-projective right $R$-modules need not be $n$-FCP-gr-projective whenever $m>n$, see Example 3.6(1).

(4) Every gr-projective right $R$-module is $n$-FCP-gr-projective.

Definition 3.5. (1) The $n$-FCP-gr-projective dimension of a graded right module $M$ is defined by

$n . \mathrm{FCP}-\mathrm{gr}-\mathrm{pd} M=\inf \left\{\mathrm{k}: \operatorname{EXT}_{R}^{k+1}\left(M, K^{n-1}\right)=0\right.$ for every special gr-copresented $\left.K^{n-1}\right\}$.

(2) The $n$-FCP-gr-projective global dimension of a graded ring $R$ is defined by r.n.FCP-gr-dim $R=\sup \{$ n.FCP-gr-pd $M \mid M$ is a graded right $R$-module $\}$.

A graded ring $R$ is called a right gr- $V$-ring if every simple graded right $R$-module is gr-injective. A graded ring $R$ is called right gr-hereditary if every submodule of a projective graded right $R$-module is projective. A graded ring $R$ is called right gr-cosemihereditary if every submodule of a projective graded right $R$-module is FCP-gr-projective, see the graded version of [21, Definition 3.6]. It is clear that gr-hereditary rings are gr-cosemihereditary.

Example 3.6. Let $R$ be a gr-cosemihereditary ring but not gr- $V$-ring, for example gr-hereditary ring $R=k[X]$ where $k$ is field. Then by graded vesion of [21, Theorem $3.9]$, there exists a graded $R$-module which is not 1-FCP-gr-projective. Also by graded vesion of [21, Theorem 3.7], r.FCP-gr-dim $R \leq 1$. So, FCP-gr-pdM $\leq 1$ for any graded right $R$-module $M$. Hence by Lemma 4.2 , there is an exact sequence $0 \rightarrow P_{1} \rightarrow P_{0} \rightarrow M \rightarrow 0$ in gr- $R$, where $P_{0}$ and $P_{1}$ are 1-FCP-gr-projective. If $U$ is a 2-copresented graded right module, then $\operatorname{EXT}_{R}^{1}\left(P_{1}, U\right)=0$, since every 2copresented graded right module is 1-copresented. Therefore from $\operatorname{EXT}_{R}^{1}\left(P_{1}, U\right) \cong$ $\operatorname{EXT}_{R}^{2}(M, U)$ we get that every graded right $R$-module $M$ is 2-FCP-gr-projective.

We have the following lemma before the next proposition.

Lemma 3.7. Let $R$ be a ring graded by a group $G, M$ a graded right $R$-module and $k$ non-negative integer. Then $\operatorname{EXT}_{R}^{k}(M,-)_{\sigma} \cong \operatorname{Ext}_{\mathrm{gr}-R}^{k}\left(M\left(\sigma^{-1}\right),-\right)$ for any $\sigma \in G$. 
Proof. It is clear that for $k=0, \operatorname{HOM}_{R}(M,-)_{\sigma} \cong \operatorname{Hom}_{\mathrm{gr}-R}\left(M\left(\sigma^{-1}\right),-\right)$, see [10]. Let $N$ be a graded right $R$-module. Then, there exists an exact sequence $0 \rightarrow N \rightarrow E \rightarrow L \rightarrow 0$, where $E$ is gr-injective. Consider the following commutative diagram with exact rows:

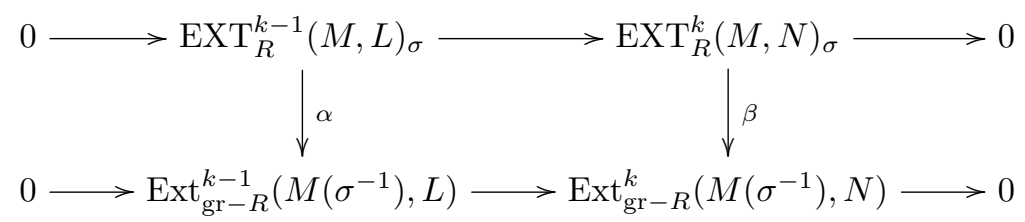

By induction hypothesis, $\alpha$ is an isomorphism and then so is $\beta$.

In the following proposition, we give some equivalent characterizations of $n$-FCPgr-projective modules with respect to special gr-copure short exact sequences.

Proposition 3.8. Let $R$ be a ring graded by a group $G$ and $n \geq 1$ an integer. Then the following statements are equivalent for a graded right $R$-module $M$.

(1) $M$ is n-FCP-gr-projective;

(2) $M$ is gr-projective with respect to all special short exact sequences in $g r-R$;

(3) The exact sequence $0 \rightarrow A \rightarrow B \rightarrow M \rightarrow 0$ in gr- $R$ is special gr-copure;

(4) $M\left(\sigma^{-1}\right)$ is n-FCP-gr-projective for any $\sigma \in G$;

(5) There exists a special gr-copure short exact sequence $0 \rightarrow K \rightarrow P \rightarrow M \rightarrow 0$ in $g r$ - $R$, where $P$ is gr-projective;

(6) There exists a special gr-copure short exact sequence $0 \rightarrow K \rightarrow P \rightarrow M \rightarrow 0$ in $g r-R$, where $P$ is $n$-FCP-gr-projective;

(7) $M\left(\sigma^{-1}\right)$ is gr-projective with respect to all special short exact sequences in gr- $R$ for any $\sigma \in G$.

Proof. (1) $\Longrightarrow$ (2) Let $0 \rightarrow K^{n-1} \rightarrow E^{n} \rightarrow K^{n} \rightarrow 0$ be a special short exact sequence with respect to any $n$-copresented graded right $R$-module $U$. Then, $\operatorname{EXT}_{R}^{1}\left(M, K^{n-1}\right) \cong \operatorname{EXT}_{R}^{n}(M, U)=0$.

$(2) \Longrightarrow(3),(3) \Longrightarrow(1),(5) \Longrightarrow(6)$ and $(6) \Longrightarrow(1)$ are clear.

$(4) \Longrightarrow(1)$ It is clear, since by Lemma 3.7, $\operatorname{EXT}_{R}^{n}(M, U)_{\sigma} \cong \operatorname{Ext}_{\mathrm{gr}-R}^{n}\left(M\left(\sigma^{-1}\right), U\right)$ for any $n$-copresented graded right $R$-module $U$ and any $\sigma \in G$.

$(1) \Longrightarrow(5)$ Let $M$ be a graded right $R$-module. Then, there is exact sequence $0 \rightarrow K \rightarrow P \rightarrow M \rightarrow 0$ in gr- $R$ with $P$ is gr-projective. By (1), $\operatorname{EXT}_{R}^{1}\left(M, K^{n-1}\right) \cong \operatorname{EXT}_{R}^{n}(M, U)=0$ for any special gr-copresented $K^{n-1}$ and any $n$-copresented graded right module $U$. So, (5) follows.

$(2) \Longleftrightarrow(7)$ It is trivial, since $\operatorname{Hom}_{\mathrm{gr}-R}\left(M\left(\sigma^{-1}\right),-\right) \cong \operatorname{HOM}_{R}(M,-)_{\sigma}$. 
$(2) \Longrightarrow(4)$ Assume that $U$ is an $n$-copresented graded right $R$-module and $0 \rightarrow K^{n-1} \rightarrow E^{n} \rightarrow K^{n} \rightarrow 0$ is a special short exact sequence in gr- $R$, where $E^{n}$ is finitely cogenerated injective. Then, we have the following exact sequence for any $\tau \in G$ :

$$
0 \longrightarrow \operatorname{HOM}_{R}\left(M, K^{n-1}\right)_{\tau} \longrightarrow \operatorname{HOM}_{R}\left(M, E^{n}\right)_{\tau} \longrightarrow \operatorname{HOM}_{R}\left(M, K^{n}\right)_{\tau} \longrightarrow 0 .
$$

Consider the following commutative diagram:

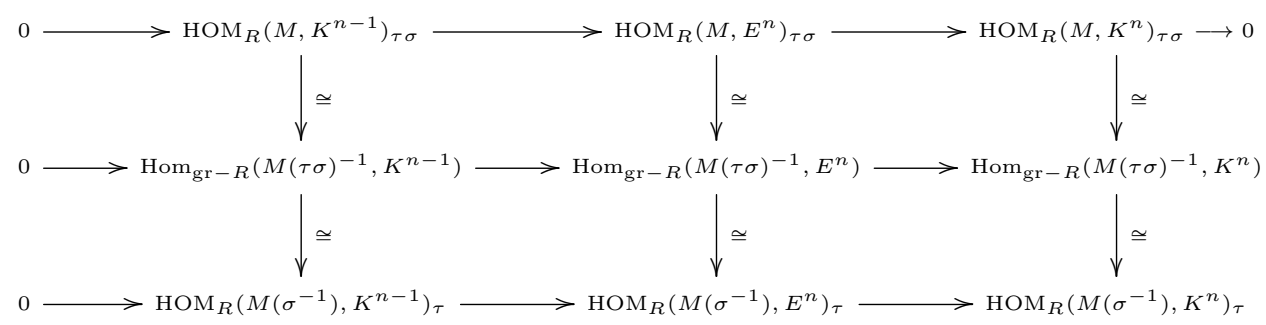

with the upper row exact for every $\tau \in G$. So, we deduce that

$0 \rightarrow \operatorname{HOM}_{R}\left(M\left(\sigma^{-1}\right), K^{n-1}\right)_{\tau} \rightarrow \operatorname{HOM}_{R}\left(M\left(\sigma^{-1}\right), E^{n}\right)_{\tau} \rightarrow \operatorname{HOM}_{R}\left(M\left(\sigma^{-1}\right), K^{n}\right)_{\tau} \rightarrow 0$

is exact, which gives rise to the exactness of

$0 \rightarrow \operatorname{HOM}_{R}\left(M\left(\sigma^{-1}\right), K^{n-1}\right) \rightarrow \operatorname{HOM}_{R}\left(M\left(\sigma^{-1}\right), E^{n}\right) \rightarrow \operatorname{HOM}_{R}\left(M\left(\sigma^{-1}\right), K^{n}\right) \rightarrow 0$.

Hence, $0=\operatorname{EXT}_{R}^{1}\left(M\left(\sigma^{-1}\right), K^{n-1}\right) \cong \operatorname{EXT}_{R}^{n}\left(M\left(\sigma^{-1}\right), U\right)$ and consequently, $M\left(\sigma^{-1}\right)$ is $n$-FCP-gr-projective.

Transfer result of $n$-FCP-gr-projective modules with respect to the functor $F$ is given in the following result.

Proposition 3.9. Let $R$ be a ring graded by a group $G$. If $M$ is an n-FCPprojective right $R$-module, then $F(M)$ is n-FCP-gr-projective.

Proof. Let $0 \longrightarrow K^{n-1} \longrightarrow E^{n} \stackrel{g}{\longrightarrow} K^{n} \longrightarrow 0$ be a special short exact sequence in gr- $R$ and $f: F(M) \rightarrow K^{n}$ a graded morphism. Since $F$ is a right adjoint functor of the forgetful functor, we have the commutative diagram:

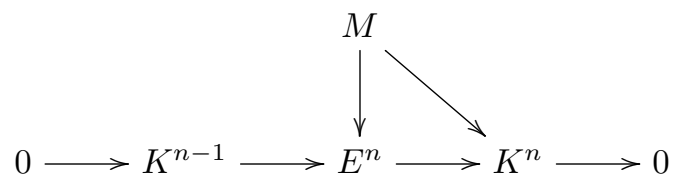


Now, again by the adjoint situation between the forgetful functor and $F$ we have a graded morphism $F(M) \rightarrow E^{n}$ such that the following diagram is commutative:

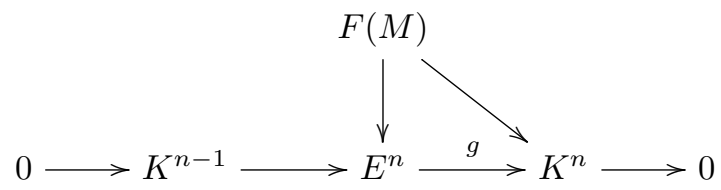

which shows that $F(M)$ is gr-projective with respect to all the special short exact sequences in gr- $R$. Let $f: F(M)\left(\sigma^{-1}\right) \rightarrow K^{n}$ be a graded morphism for any $\sigma \in G$. Since, the exact sequence

$$
0 \longrightarrow K^{n-1}(\sigma) \longrightarrow E^{n}(\sigma) \stackrel{T_{\sigma}(g)}{\longrightarrow} K^{n}(\sigma) \longrightarrow 0
$$

exists and $K^{n-1}(\sigma)$ is special gr-copresented, there exists a graded morphism $h$ : $F(M) \rightarrow E^{n}(\sigma)$ such that $T_{\sigma}(g) h=T_{\sigma}(f)$, and so $g T_{\sigma^{-1}}(h)=f$ for $T_{\sigma^{-1}}(h):$ $F(M)\left(\sigma^{-1}\right) \rightarrow E^{n}$. Therefore for any $\sigma \in G, F(M)\left(\sigma^{-1}\right)$ is gr-projective with respect to all the special short exact sequences and consequently by Proposition $3.8, F(M)$ is $n$-FCP-gr-projective.

Also, as for the classical projective notion, the class $g r-\mathcal{F C P} \mathcal{P}_{n}$ is closed under direct limits.

Proposition 3.10. Let $R$ be a graded ring by a group $G, K^{n-1}$ a special grcopresented and $\left\{M_{i}\right\}_{i \in I}$ a direct system of graded right $R$-modules with I directed.

Then:

(1) $\operatorname{HOM}_{R}\left(\lim _{\longrightarrow} M_{i}, K^{n-1}\right) \cong \lim _{\leftarrow} \operatorname{HOM}_{R}\left(M_{i}, K^{n-1}\right)$.

(2) $\operatorname{EXT}_{R}^{1}\left(\lim _{\longrightarrow} M_{i}, K^{n-1}\right) \cong \lim _{\leftarrow} \operatorname{EXT}_{R}^{1}\left(M_{i}, K^{n-1}\right)$.

Proof. (1) For any $\sigma \in G$, we have

$\operatorname{HOM}_{R}\left(\lim _{\rightarrow} M_{i}, K^{n-1}\right)=\bigoplus_{\sigma \in G} \operatorname{HOM}_{R}\left(\lim _{\rightarrow} M_{i}, K^{n-1}\right)_{\sigma} \cong \underset{\sigma \in G}{\bigoplus} \operatorname{Hom}_{\mathrm{gr}-R}\left(\lim _{\rightarrow} M_{i}\left(\sigma^{-1}\right), K^{n-1}\right)$.

By [17, Proposition 5.26],

$$
\bigoplus_{\sigma \in G} \operatorname{Hom}_{\mathrm{gr}-R}\left(\lim _{-} M_{i}\left(\sigma^{-1}\right), K^{n-1}\right) \cong \lim _{\leftarrow} \bigoplus_{\sigma \in G} \operatorname{Hom}_{\mathrm{gr}-R}\left(M_{i}\left(\sigma^{-1}\right), K^{n-1}\right) .
$$

So,

$\lim _{\leftarrow} \bigoplus_{\sigma \in G} \operatorname{Hom}_{\mathrm{gr}-R}\left(M_{i}\left(\sigma^{-1}\right), K^{n-1}\right) \cong \lim _{\leftarrow} \bigoplus_{\sigma \in G} \operatorname{HOM}_{R}\left(M_{i}, K^{n-1}\right)_{\sigma}=\lim _{\leftarrow} \operatorname{HOM}_{R}\left(M_{i}, K^{n-1}\right)$. 
(2) Let $0 \rightarrow K^{n-1} \rightarrow E^{n} \rightarrow K^{n} \rightarrow 0$ be a special short exact sequence in gr- $R$.

Then by (1), the following commutative diagram exists:
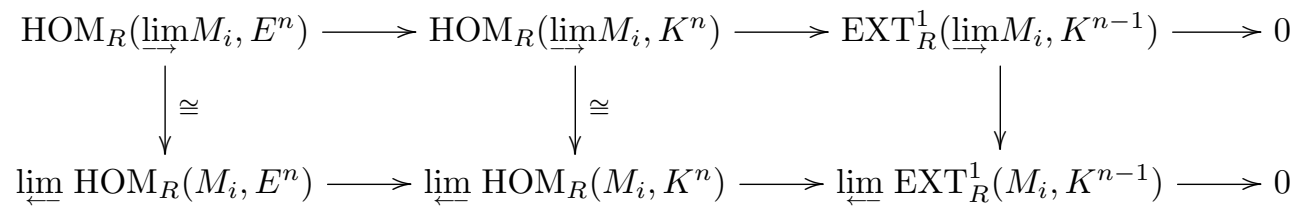

Therefore, $\operatorname{EXT}_{R}^{1}\left(\lim _{\cong} M_{i}, K^{n-1}\right) \cong \lim \operatorname{EXT}_{R}^{1}\left(M_{i}, K^{n-1}\right)$.

Corollary 3.11. Let $R$ be a graded ring. Then, the class gr- $\mathcal{F C P}{ }_{n}$ is closed under direct limits.

Proof. Let $U$ be an $n$-copresented graded right module and let $\left\{M_{i}\right\}_{i \in I}$ be a family of $n$-FCP-gr-projective right modules . Then by Proposition 3.10,

$\operatorname{EXT}_{R}^{n}\left(\lim _{\longrightarrow} M_{i}, U\right) \cong \operatorname{EXT}_{R}^{1}\left(\lim _{\longrightarrow} M_{i}, K^{n-1}\right) \cong \lim _{\leftarrow} \operatorname{EXT}_{R}^{1}\left(M_{i}, K^{n-1}\right) \cong \lim _{\leftarrow} \operatorname{EXT}_{R}^{n}\left(M_{i}, U\right)$, where $K^{n-1}$ is special gr-copresented.

\section{4. n-gr-cocoherent rings}

In this section, some characterizations of $n$-FCP-gr-projective right modules on right $n$-gr-cocoherent rings are given.

We state the following lemmas that are derived from [21, Theorem 2.12].

Lemma 4.1. Let $R$ be a right n-gr-cocoherent ring and $M$ a graded right $R$-module.

Then the following statements are equivalent:

(1) $n . F C P-g r-p d M \leq k$;

(2) $\operatorname{EXT}_{R}^{k+1}\left(M, K^{n-1}\right)=0$ for any special gr-copresented $K^{n-1}$.

Proof. $(2) \Longrightarrow(1)$ is trivial by Definition 3.5.

$(1) \Longrightarrow(2)$ Use induction on $k$. Clear if $n$.FCP-gr-pd $M=k$. Let $n$.FCP$\operatorname{gr}-\operatorname{pd} M \leq k-1$. If $0 \rightarrow K^{n-1} \rightarrow E^{n} \rightarrow K^{n} \rightarrow 0$ is a special short exact sequence in gr- $R$ with respect to any $n$-copresented graded right $R$-module $U$, then from the $n$-gr-cocoherence of $R$ we deduce that $K^{n}$ is special gr-copresented. Also, we have $\operatorname{EXT}_{R}^{k}\left(M, K^{n}\right) \cong \operatorname{EXT}_{R}^{k+1}\left(M, K^{n-1}\right)$. So by induction hypothesis, $\operatorname{EXT}_{R}^{k}\left(M, K^{n}\right)=0$ and consequently $\operatorname{EXT}_{R}^{k+1}\left(M, K^{n-1}\right)=0$ which completes the proof.

Lemma 4.2. Let $R$ be a right n-gr-cocoherent ring, $M$ a graded right $R$-module and $k$ a non-negative integer. Then the following statements are equivalent:

(1) $n . F C P-g r-p d M \leq k$; 
(2) $\operatorname{EXT}_{R}^{k+l}\left(M, K^{n-1}\right)=0$ for any special gr-copresented $K^{n-1}$ and all positive integers $l$;

(3) $\operatorname{EXT}_{R}^{k+1}\left(M, K^{n-1}\right)=0$ for any special gr-copresented $K^{n-1}$;

(4) There exists an exact sequence

$$
0 \longrightarrow P_{k} \stackrel{f_{k}}{\longrightarrow} P_{k-1} \stackrel{f_{k-1}}{\longrightarrow} \cdots P_{1} \stackrel{f_{1}}{\longrightarrow} P_{0} \stackrel{f_{0}}{\longrightarrow} M \longrightarrow 0
$$

in gr- $R$ with $P_{0}, P_{1}, \cdots, P_{k}$ are $n$-FCP-gr-projective;

(5) n.FCP-gr-pdM $\left(\sigma^{-1}\right) \leq k$ for any $\sigma \in G$.

Proof. (1) $\Longrightarrow$ (2) If $n$.FCP-gr-pd $M \leq k$, then $n$.FCP-gr-pd $M \leq k+l-1$. So by Lemma 4.1, $\operatorname{EXT}_{R}^{k+l}\left(M, K^{n-1}\right)=0$.

$(4) \Longrightarrow(1)$ Since $R$ is right $n$-gr-cocoherent, by Lemma $4.1, \operatorname{EXT}_{R}^{j}\left(P_{i}, K^{n-1}\right)=$ 0 for any special gr-copresented $K^{n-1}$, all positive integers $j$ and any $0 \leq i \leq k$. So by (4), we have:

$$
\operatorname{EXT}_{R}^{k+1}\left(M, K^{n-1}\right) \cong \operatorname{EXT}_{R}^{k}\left(k e r\left(f_{0}\right), K^{n-1}\right) \cong \ldots \cong \operatorname{EXT}_{R}^{1}\left(P_{k}, K^{n-1}\right) .
$$

Hence by Lemma 4.1, $n$.FCP-gr-pd $M \leq k$.

$(2) \Longrightarrow(3)$ It is obvious.

$(3) \Longrightarrow(4)$ For every graded right $R$-module $M$, there exists an exact sequence

$$
0 \longrightarrow P_{k} \longrightarrow P_{k-1} \longrightarrow \cdots P_{1} \longrightarrow P_{0} \longrightarrow M \longrightarrow 0
$$

in gr- $R$ with $P_{0}, P_{1}, \cdots, P_{k-1}$ are gr-projective. Therefore for any positive integers $l$, we have $\operatorname{EXT}_{R}^{l}\left(P_{i}, K^{n-1}\right)=0$ for all special gr-copresented modules $K^{n-1}$ and any $0 \leq i \leq k-1$. Let $K_{i}=\operatorname{ker}\left(P_{i} \rightarrow P_{i-1}\right)$. Then,

$\operatorname{EXT}_{R}^{k+1}\left(M, K^{n-1}\right) \cong \operatorname{EXT}_{R}^{k}\left(K_{0}, K^{n-1}\right) \cong \operatorname{EXT}_{R}^{k-1}\left(K_{1}, K^{n-1}\right) \cong \operatorname{EXT}_{R}^{1}\left(P_{k}, K^{n-1}\right)$.

By (3), $\operatorname{EXT}_{R}^{k+1}\left(M, K^{n-1}\right)=0$, and so $\operatorname{EXT}_{R}^{1}\left(P_{k}, K^{n-1}\right)=0$, which means that $P_{k}$ is $n$-FCP-gr-projective.

$(1) \Longleftrightarrow(5)$ Use induction on $k$. If $k=0$, then by Proposition $3.8, M$ is $n$-FCPgr-projective if and only if $M\left(\sigma^{-1}\right)$ is $n$-FCP-gr-projective for any $\sigma \in G$. Assume that $k>0$. There is an exact sequence $0 \rightarrow L \stackrel{f}{\rightarrow} P \rightarrow M \rightarrow 0$ in gr- $R$, where $P$ is gr-projective. For any $\sigma \in G$, it follows that the exact sequence

$$
0 \longrightarrow L\left(\sigma^{-1}\right) \stackrel{T_{\sigma^{-1}}(f)}{\longrightarrow} P\left(\sigma^{-1}\right) \longrightarrow M\left(\sigma^{-1}\right) \longrightarrow 0
$$

where $P\left(\sigma^{-1}\right)$ is gr-projective exists. So by Lemma 3.7, for every special $g r$ copresented $K^{n-1}$ and any $\tau \in G$, we have:

$\operatorname{EXT}_{R}^{k+1}\left(M\left(\sigma^{-1}\right), K^{n-1}\right)_{\tau} \cong \operatorname{EXT}_{R}^{k}\left(L\left(\sigma^{-1}\right), K^{n-1}\right)_{\tau} \cong \operatorname{Ext}_{\mathrm{gr}-R}^{k}\left(L(\tau \sigma)^{-1}, K^{n-1}\right) \cong$ 
$\operatorname{EXT}_{R}^{k}\left(L, K^{n-1}\right)_{\tau \sigma} \cong \operatorname{EXT}_{R}^{k+1}\left(M, K^{n-1}\right)_{\tau \sigma}$. By induction hypothesis, $n$.FCP$\operatorname{gr}-\operatorname{pd} L\left(\sigma^{-1}\right) \leq k-1$ if and only if $n$.FCP-gr-pd $L \leq k-1$. So, we deduce that $n$.FCP-gr-pd $M\left(\sigma^{-1}\right) \leq k$ if and only if $n$.FCP-gr-pd $M \leq k$.

Corollary 4.3. Let $R$ be a right n-gr-cocoherent ring of type $G$. Then the following statements are equivalent:

(1) If $\phi: N \rightarrow M$ is an $n$-FCP-gr-projective preenvelope, then $N$ has an epic n-FCP-gr-projective preenvelope;

(2) The cokernel of any $n$-FCP-gr-projective preenvelope of a graded right $R$ module is n-FCP-gr-projective;

Moreover, if every submodule of an n-FCP-gr-projective graded right $R$ module has an n-FCP-gr-projective preenvelope, the above are equivalent to:

(3) r.n.FCP-gr-dimR $\leq 1$.

Proof. (1) $\Longrightarrow(2)$ Let $\phi: N \rightarrow M$ be an $n$-FCP-gr-projective preenvelope. Then $f: \operatorname{Im}(\phi) \rightarrow M$ is an $n$-FCP-gr-projective preenvelope. By (1), there is an epic $n$ FCP-gr-projective preenvelope $g: \operatorname{Im}(\phi) \rightarrow C$. Consider the following commutative diagram, where $D$ is a pushout of two maps $f$ and $g$ :

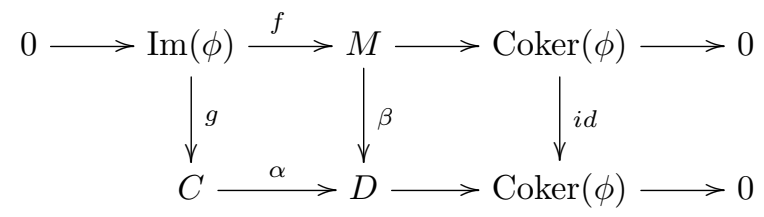

By [17, Exercise 5.10], $\alpha$ is injective and $\beta$ is surjective. On the other hand, $D=$ $\alpha(C)+\beta(M)$. Since $\beta$ is surjective, $D=\alpha(C)+D$ and so, $\alpha(C) \subseteq D$. Also, by using of preenvelopes $f$ and $g$, there is a graded morphism $h: D \rightarrow C$ such that $h \alpha=1_{C}$. Hence, $D \cong C \oplus \operatorname{Coker}(\phi)$. Similarly $D \cong M$. Therefore from $n$-FCP-gr-projectivity $M$, we deduce that $\operatorname{Coker}(\phi)$ is $n$-FCP-gr-projective.

(2) $\Longrightarrow(1)$ Let $\phi: N \rightarrow M$ be an $n$-FCP-gr-projective preenvelope. It is enough to show that $\operatorname{Im}(\phi)$ is $n$-FCP-gr-projective. Consider the exact sequence $0 \rightarrow$ $\operatorname{Im}(\phi) \rightarrow M \rightarrow \operatorname{Coker}(\phi) \rightarrow 0$. By hypothesis, Coker $(\phi)$ is $n$-FCP-gr-projective, and so for every special gr-copresented $K^{n-1}$, we have:

$$
0=\operatorname{EXT}_{R}^{1}\left(M, K^{n-1}\right) \longrightarrow \operatorname{EXT}_{R}^{1}\left(\operatorname{Im}(\phi), K^{n-1}\right) \longrightarrow \operatorname{EXT}_{R}^{2}\left(\operatorname{Coker}(\phi), K^{n-1}\right) .
$$

Hence by Lemm 4.2 and $(2), \operatorname{EXT}_{R}^{2}\left(\operatorname{Coker}(\phi), K^{n-1}\right)=0$. Hence,

$$
0=\operatorname{EXT}_{R}^{1}\left(\operatorname{Im}(\phi), K^{n-1}\right) \cong \operatorname{EXT}_{R}^{n}(\operatorname{Im}(\phi), U)
$$


for any $n$-copresented graded right module $U$, and then it follows that $\operatorname{Im}(\phi)$ is $n$-FCP-gr-projective.

(2) $\Longrightarrow(3)$ Let $M$ be a graded right $R$-module. Then, there exists an exact sequence $0 \rightarrow K \rightarrow P \rightarrow M \rightarrow 0$ in gr- $R$, where $P$ is gr-projective. If $K$ is $n$-FCP-gr-projective, then r.n.FCP-gr-dim $(R) \leq 1$. So, we show that $K$ is $n$-FCPgr-projective. If $\phi: K \rightarrow N$ is an $n$-FCP-gr-projective preenvelope, then $\phi$ is injective. So similar to proof of $(2) \Longrightarrow(1)$, we get that $K$ is $n$-FCP-gr-projective.

(3) $\Longrightarrow$ (1) Let $\phi: N \rightarrow M$ be an $n$-FCP-gr-projective preenvelope. Then by Lemma 4.2, the exact sequence $0 \rightarrow \operatorname{Im}(\phi) \rightarrow M \rightarrow \operatorname{Coker}(\phi) \rightarrow 0$ implies that $\operatorname{Im}(\phi)$ is $n$-FCP-gr-projective, and so $N \rightarrow \operatorname{Im}(\phi)$ is an epic $n$-FCP-gr-projective preenvelope of $N$.

In the following, we present one of the main results in this paper.

Theorem 4.4. Let $R$ be a graded ring. Then the following statements are equivalent:

(1) $R$ is right $n$-gr-cocoherent;

(2) For every exact sequence $0 \rightarrow A \rightarrow B \rightarrow C \rightarrow 0$ in $g r-R, A$ is n-FCP-grprojective if $B$ and $C$ are $n-F C P$-gr-projective.

Proof. (1) $\Longrightarrow(2)$ Let $U$ be an $n$-copresented graded right $R$-module. Then by Lemm 4.2(2), we have: $0=\operatorname{EXT}_{R}^{n}(B, U) \rightarrow \operatorname{EXT}_{R}^{n}(A, U) \rightarrow \operatorname{EXT}_{R}^{n+1}(C, U)=0$, since $B$ and $C$ are $n$-FCP-gr-projective. So $\operatorname{EXT}_{R}^{n}(A, U)=0$, and hence $A$ is $n$-FCP-gr-projective.

(2) $\Longrightarrow$ (1) Let $U$ be an $n$-copresented graded right $R$-module and $0 \rightarrow K^{n-1} \rightarrow$ $E^{n} \rightarrow K^{n} \rightarrow 0$ a special short exact sequence in $\operatorname{gr}-R$ with respect to $U$. We show that $U$ is $(n+1)$-copresented. For this, enough to say that $K^{n}$ is special gr-copresented. Let $M$ be a 1-FCP-projective right $R$-module and $0 \rightarrow K \rightarrow P \rightarrow$ $M \rightarrow 0$ an exact sequence in Mod- $R$ with $P$ is projective. Then $0 \rightarrow F(K) \rightarrow$ $F(P) \rightarrow F(M) \rightarrow 0$ is exact in gr- $R$, where by Proposition 3.9, $F(P)$ and $F(M)$ are 1-FCP-gr-projective. Hence, Remark 3.4 imply that $F(P)$ and $F(M)$ are $n$ FCP-gr-projective. So by hypothesis, it follows that $F(K)$ is $n$-FCP-gr-projective. Since $E^{n}$ is $g r$-injective, we have:

$0=\operatorname{Ext}_{\mathrm{gr}-R}^{1}\left(F(M), E^{n}\right) \longrightarrow \operatorname{Ext}_{\mathrm{gr}-R}^{1}\left(F(M), K^{n}\right) \longrightarrow \operatorname{Ext}_{\mathrm{gr}-R}^{2}\left(F(M), K^{n-1}\right) \longrightarrow 0$. So, $\operatorname{Ext}_{\mathrm{gr}-R}^{1}\left(F(M), K^{n}\right) \cong \operatorname{Ext}_{\mathrm{gr}-R}^{2}\left(F(M), K^{n-1}\right)$. Also, we have:

$0=\operatorname{Ext}_{\mathrm{gr}-R}^{1}\left(F(P), K^{n-1}\right) \rightarrow \operatorname{Ext}_{\mathrm{gr}-R}^{1}\left(F(K), K^{n-1}\right) \rightarrow \operatorname{Ext}_{\mathrm{gr}-R}^{2}\left(F(M), K^{n-1}\right) \rightarrow 0$.

Consequently $\operatorname{Ext}_{\mathrm{gr}-R}^{1}\left(F(K), K^{n-1}\right) \cong \operatorname{Ext}_{\mathrm{gr}-R}^{2}\left(F(M), K^{n-1}\right)$ and so by Lemma 3.7, $n$-FCP-gr-projectivity $F(K)$ imply that $\operatorname{Ext}_{\mathrm{gr}-R}^{1}\left(F(K)\left(\sigma^{-1}\right), K^{n-1}\right)=0$ for 
any $\sigma \in G$. Thus,

$$
0=\operatorname{Ext}_{\mathrm{gr}-R}^{1}\left(F(K), K^{n-1}\right) \cong \operatorname{Ext}_{\mathrm{gr}-R}^{2}\left(F(M), K^{n-1}\right) \cong \operatorname{Ext}_{\mathrm{gr}-R}^{1}\left(F(M), K^{n}\right) .
$$

Now, consider the following commutative diagram:

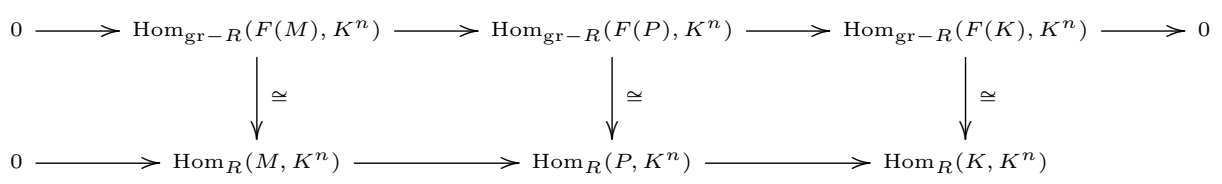

with the upper row exact. Therefore, $\operatorname{Ext}_{\mathrm{gr}-R}^{1}\left(F(M), K^{n}\right) \cong \operatorname{Ext}_{R}^{1}\left(M, K^{n}\right)=0$ for any 1-FCP-projective $R$-module $M$, and it follows that $K^{n}$ is 1-copresented. Hence, $U$ is $(n+1)$-copresented.

Corollary 4.5. Let $R$ be a right n-gr-cocoherent ring. Then, graded right $R$-module $M$ is n-FCP-gr-projective if and only if every copure epimorphic image and copure submodule of $M$ is n-FCP-gr-projective.

Proof. $(\Longrightarrow)$ Let $N$ be a copure submodule of $n$-FCP-gr-projective right $R$-module $M$. Then, the exact sequence $0 \rightarrow N \rightarrow M \rightarrow \frac{M}{N} \rightarrow 0$ is special gr-copure. So by Proposition 3.8, $\frac{M}{N}$ is $n$-FCP-gr-projective and hence by Theorem $4.4, N$ is $n$-FCP-gr-projective.

$(\Longleftarrow)$ It is clear.

Before the next results, we first introduce the following symbols and definitions given in $[9,20]$.

For every class $\mathcal{Y}$ of graded right $R$-modules, denote the classes

$$
\mathcal{Y}^{\perp}=\left\{X \in g r-R: \operatorname{Ext}_{\mathrm{gr}-R}^{1}(Y, X)=0 \text { for all } Y \in \mathcal{Y}\right\}
$$

and

$$
{ }^{\perp} \mathcal{Y}=\left\{X \in g r-R: \operatorname{Ext}_{\mathrm{gr}-R}(X, Y)=0 \text { for all } Y \in \mathcal{Y}\right\} .
$$

Given two classes of graded right $R$-modules $\mathcal{F}$ and $\mathcal{C}$, then we say that $(\mathcal{F}, \mathcal{C})$ is a cotorsion theory in gr- $R$ if $\mathcal{F}^{\perp}=\mathcal{C}$ and $\mathcal{F}={ }^{\perp} \mathcal{C}$. A cotorsion theory $(\mathcal{F}, \mathcal{C})$ is called hereditary if whenever $0 \rightarrow F^{\prime} \rightarrow F \rightarrow F^{\prime \prime} \rightarrow 0$ is exact in gr- $R$ with $F, F^{\prime \prime} \in \mathcal{F}$ then $F^{\prime}$ is also in $\mathcal{F}$.

A duality pair over a graded $\operatorname{ring} R$ is a pair $(\mathcal{F}, \mathcal{C})$, where $\mathcal{F}$ is a class of graded right (resp. left) $R$-modules and $\mathcal{C}$ is a class of graded left (resp. right) $R$-modules, subject to the following conditions: (1) For any graded module $F$, one has $F \in \mathcal{F}$ if and only if $F^{*} \in \mathcal{C}$. (2) $\mathcal{C}$ is closed under direct summands and finite direct sums.

A duality pair $(\mathcal{F}, \mathcal{C})$ is called (co)product-closed if the class of $\mathcal{F}$ is closed under graded direct (co)products, and a duality pair $(\mathcal{F}, \mathcal{C})$ is called perfect if it is coproduct-closed, $\mathcal{F}$ is closed under extensions and $R$ belongs to $\mathcal{F}$. 
Theorem 4.6. The pair $\left(g r-\mathcal{F C P}{ }_{n}, g r-\mathcal{F C P}{ }_{n}^{\perp}\right)$ is hereditary cotorsion theory if and only if $R$ is a right $n$-gr-cocoherent ring.

Proof. $(\Longrightarrow)$ Let $M$ be an $n$-FCP-gr-projective right $R$-module. Then, there is an exact sequence $0 \rightarrow K \rightarrow P \rightarrow M \rightarrow 0$ in gr- $R$, where $P$ is gr-projective. Thus by Remark 3.4, $P$ is $n$-FCP-gr-projective, too. Since $\left(g r-\mathcal{F C P}{ }_{n}, g r-\mathcal{F C P}{ }_{n}^{\perp}\right)$ is a hereditary cotorsion theory, we deduce that $K$ is $n$-FCP-gr-projective, and then by Theorem 4.4, it follows that $R$ is right $n$-gr-cocoherent.

$(\Longleftarrow)$ Note that we have to show that ${ }^{\perp}\left(g r-\mathcal{F C P}{ }_{n}^{\perp}\right)=g r-\mathcal{F C P}{ }_{n}$. Let $K^{n-1}$ be a special gr-copresented with respect to any $n$-copresented graded right $R$-module $U$ and $M \in{ }^{\perp}\left(g r-\mathcal{F C P}{ }_{n}^{\perp}\right)$. Then, $K^{n-1} \in g r-\mathcal{F C P}{ }_{n}^{\perp}$ and $M\left(\sigma^{-1}\right) \in \in^{\perp}\left(g r-\mathcal{F C P}{ }_{n}^{\perp}\right)$ for all $\sigma \in G$ by analogy with the proof of Proposition 3.9. Therefore by Lemma 3.7, $\operatorname{EXT}_{R}^{1}\left(M, K^{n-1}\right)_{\sigma} \cong \operatorname{Ext}_{\mathrm{gr}-R}^{1}\left(M\left(\sigma^{-1}\right), K^{n-1}\right)=0$ and consequently by Lemma 4.2, $M$ is $n$-FCP-gr-projective, and hence $M \in g r-\mathcal{F C P}_{n}$. Let $0 \rightarrow F^{\prime} \rightarrow F \rightarrow$ $F^{\prime \prime} \rightarrow 0$ be a exact sequence of modules in gr- $R$, where $F$ and $F^{\prime \prime}$ are $n$-FCPgr-projective. Then by Theorem $4.4, F^{\prime}$ is $n$-FCP-gr-projective, since $R$ is $n$-grcocoherent. So, it follows that $\left(g r-\mathcal{F C P}{ }_{n}, g r-\mathcal{F C P}{ }_{n}^{\perp}\right)$ is a hereditary cotorsion theory.

We denote $\left.(g r-\mathcal{F} \mathcal{C P})_{n}\right)^{*}=\left\{M^{*} \mid M \in g r-\mathcal{F C P} \mathcal{P}_{n}\right\}$. The following lemma shows the connection between $n$-FCP-gr-projective and $n$-FP-gr-injective modules.

Lemma 4.7. Let $R$ be a graded ring of type $G$.

(1) If $U$ is an n-presented graded left $R$-module, then $U^{*}$ is an $n$-copresented graded right $R$-module.

(2) $(g r-\mathcal{F C P})^{*} \subseteq g r-\mathcal{F} \mathcal{I}_{n}$.

Proof. (1) Let $U$ be an $n$-presented graded left $R$-module. Then, there exists an exact sequence

$$
F_{n} \longrightarrow F_{n-1} \longrightarrow \cdots \longrightarrow F_{0} \longrightarrow U \longrightarrow 0
$$

in $R$-gr with each $F_{i}$ is finitely generated free. So by graded version of [17, Lemma $3.53]$, there is an exact sequence

$$
0 \longrightarrow U^{*} \longrightarrow F_{0}^{*} \longrightarrow \cdots \longrightarrow F_{n-1}^{*} \longrightarrow F_{n}^{*}
$$

with $R$-modules in gr- $R$. It suffices to show that every $F_{i}^{*}$ is finitely cogenerated injective. It is clear that any $F_{i}^{*}$ is finitely cogenerated. So, we prove that every $F_{i}^{*}$ is injective, too. Consider the short exact sequence $0 \rightarrow A \rightarrow B \rightarrow C \rightarrow 0$ in gr- $R$. Then, there exists the following commutative diagram with the upper row 
exact for any $\sigma \in G$ :

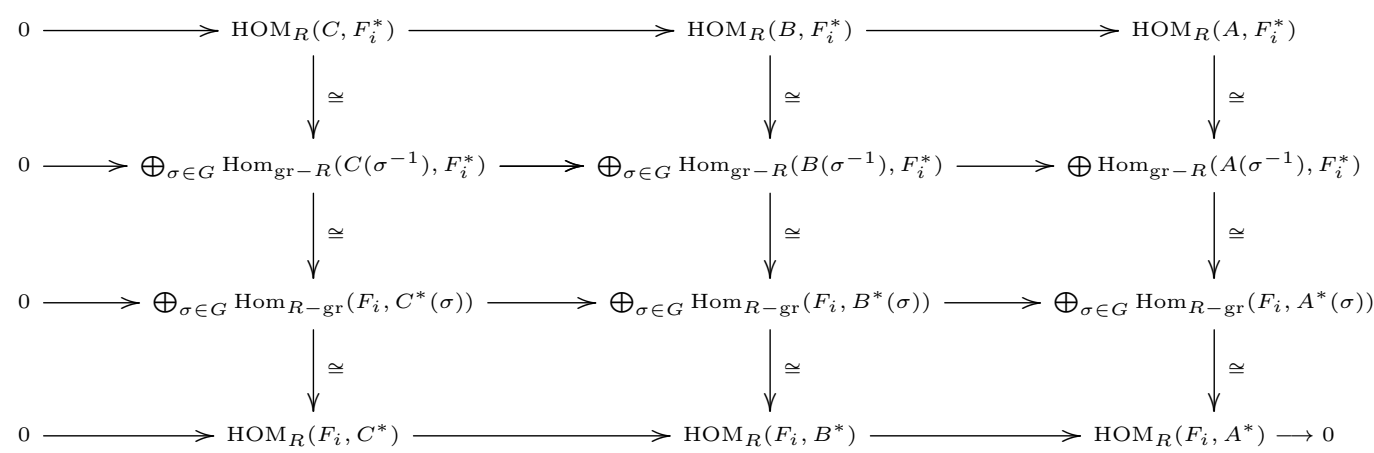

So, $\operatorname{EXT}_{R}^{1}\left(C, F_{i}^{*}\right)=0$ and hence any $F_{i}^{*}$ is injective.

(2) By [20, Definition 3.1], let $0 \rightarrow K_{n-1} \rightarrow P_{n-1} \rightarrow K_{n} \rightarrow 0$ be a special short exact sequence in $R$-gr with respect to any $n$-presented graded left $R$-module $U$. Then by (1), $K_{n-1}^{*}$ is special gr-copresented in gr- $R$. So if $M$ is $n$-FCP-grprojective right $R$-module, then similar to the proof (1), $0=\operatorname{EXT}_{R}^{1}\left(M, K_{n-1}^{*}\right) \cong$ $\operatorname{EXT}_{R}^{1}\left(K_{n-1}, M^{*}\right)$. On the other hand, $\operatorname{EXT}_{R}^{n}\left(U, M^{*}\right) \cong \operatorname{EXT}_{R}^{1}\left(K_{n-1}, M^{*}\right)$ by $[20$, Definition 3.1]. Therefore, $M^{*}$ is an $n$-FP-gr-injective left $R$-module, and then we conclude that $\left.(g r-\mathcal{F C P})_{n}\right)^{*} \subseteq g r-\mathcal{F} \mathcal{I}_{n}$.

In the following theorem, by using the previous results, we present some equivalent characterizations to that each graded right $R$-module is $n$-FCP-gr-projective.

Theorem 4.8. Let $R$ be a graded ring of type $G$. Then, the following statements are equivalent:

(1) Every graded right module is $n$-FCP-gr-projective;

(2) $\operatorname{gr}$-id $(U) \leq n-1$ for any $n$-copresented graded right $R$-module $U$;

(3) Every special gr-copresented right module is gr-injective;

(4) $\left(g r-\mathcal{F C P}{ }_{n}, g r-\mathcal{F C P}{ }_{n}^{\perp}\right)$ is perfect hereditary cotorsion theory and $N$ has an $n$-FCP-gr-projective cover with the unique mapping property for any $N \in$ $\operatorname{gr}-\mathcal{F} \mathcal{C P}{ }_{n}^{\perp}$

(5) $N$ is gr-injective for any $N \in g r-\mathcal{F C P}{ }_{n}^{\perp}$;

(6) $N(\sigma)$ is gr-injective for any $N \in$ gr- $\mathcal{F} \mathcal{C} \mathcal{P}_{n}^{\perp}$ and any $\sigma \in G$;

(7) Every graded right module has an $n$-FCP-gr-projective cover with the unique mapping property;

(8) $R$ is right $n$-gr-cocoherent and $N$ is $n$-FCP-gr-projective for any $N \in g r$ $\mathcal{F C P}{ }_{n}^{\perp}$.

Proof. $(1) \Longrightarrow(2),(2) \Longrightarrow(3)$ and $(3) \Longrightarrow(1)$ are clear by Proposition 3.8.

$(1) \Longrightarrow(5)$ and $(5) \Longrightarrow(3)$ are obvious. 
$(5) \Longleftrightarrow(6)$ it follows from $\operatorname{HOM}_{R}(-, N)_{\sigma} \cong \operatorname{Hom}_{\mathrm{gr}-R}(-, N(\sigma))$.

$(1) \Longrightarrow(7)$ First, we show that the class $g r-\mathcal{F C P} \mathcal{P}_{n}$ is covering. If $M \in g r$ $\mathcal{F C P}{ }_{n}$, then by Lemma $4.7, M^{*} \in g r-\mathcal{F P}{ }_{n}$. Contrary, if $M^{*} \in g r-\mathcal{F} \mathcal{P}_{n}$, then $[20$, Proposition 3.8] implies that $M$ is $n$-gr-flat, and hence by (1), $M \in g r-\mathcal{F C P}{ }_{n}$. On the other hand, the class $g r-\mathcal{F} \mathcal{P}_{n}$ is closed under direct summands and direct sums by [20, Propositions 3.7 and 3.16]. So, we obtain that $\left(g r-\mathcal{F C P}{ }_{n}, g r-\mathcal{F} \mathcal{I}_{n}\right)$ is a duality pair. Also By (1), it follows that the class $g r-\mathcal{F C P} \mathcal{P}_{n}$ is closed under gr-pure submodules, gr-pure quotients and gr-pure extensions. Therefore by Corollary 3.11 and [20, Theorem 4.2], the class $g r-\mathcal{F C P}{ }_{n}$ is covering and hence by hypothesis, (7) follows.

$(7) \Longrightarrow(1)$ Let $N$ be a graded right $R$-module. Then there is a commutative diagram with exact rows:

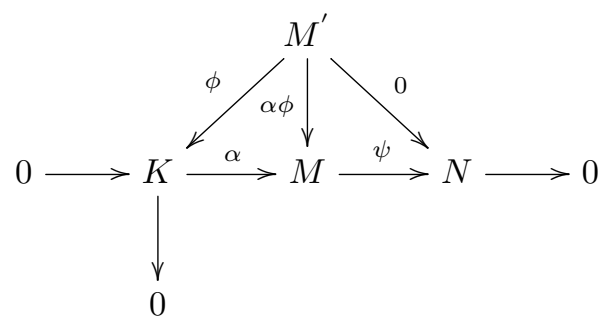

where, $\psi$ and $\phi$ are $n$-FCP-gr-projective cover with the unique mapping property. Since $\psi \alpha \phi=0=\psi$, we have $\alpha \phi=0$ by (7). Therefore, $K=\operatorname{im}(\phi) \subseteq \operatorname{ker}(\alpha)=0$ and so $K=0$. Thus $N \cong M$ and hence every graded right module $N$ is $n$-FCP-grprojective.

(1) $\Longrightarrow(8)$ Let $M$ be an $n$-FCP-gr-projective right $R$-module. Then, there is an exact sequence $0 \rightarrow K \rightarrow P \rightarrow M \rightarrow 0$ in gr- $R$, where $P$ and $K$ are $n$-FCPgr-projective by (1). So by Theorem 4.4, it follows that $R$ is right $n$-gr-cocoherent. Also by hypothesis, $N$ is $n$-FCP-gr-projective for any $N \in g r-\mathcal{F C P}{ }_{n}^{\perp}$.

$(8) \Longrightarrow(3)$ Cosider the special short exact sequence $\Delta^{\prime}: 0 \rightarrow K^{n-1} \rightarrow E^{n-1} \rightarrow$ $K^{n} \rightarrow 0$ in gr- $R$ with respect to any $n$-copresented graded right module $U$, where $K^{n-1}$ is gr-copresented and $K^{n}$ is $n$-gr-cogenerated. But $K_{n}$ is gr-copresented, too, since $R$ is $n$-gr-cocoherent. Thus $K^{n} \in g r-\mathcal{F C P}{ }_{n}^{\perp}$ and consequently $0=$ $\operatorname{EXT}_{R}^{n}\left(K^{n}, U\right) \cong \operatorname{EXT}_{R}^{1}\left(K^{n}, K^{n-1}\right)$, since $K_{n}$ is $n$-FCP-gr-projective by (8). Therefore $\Delta^{\prime}$ is split and we deduce that $K^{n-1}$ is gr-injective.

(4) $\Longrightarrow(8)$ By Theorem 4.6, $R$ is right $n$-gr-cocoherent. Let $N \in g r-\mathcal{F C P}{ }_{n}^{\perp}$. If $\phi: M \rightarrow N$ is an $n$-FCP-gr-projective cover with the unique mapping property, then $\operatorname{ker} \phi \in g r-\mathcal{F C P}{ }_{n}^{\perp}$. Thus, similar to the proof of $(7) \Longrightarrow(1)$, we get that $N$ is $n$-FCP-gr-projective. 
$(8) \Longrightarrow(4)$ By Theorem 4.6, $\left(g r-\mathcal{F C P}{ }_{n}, g r-\mathcal{F C P}{ }_{n}^{\perp}\right)$ is hereditary cotorsion theory. Also $R \in g r-\mathcal{F C P} \mathcal{P}_{n}$ and by Corollary 3.11 and $(8) \Longrightarrow(3) \Longrightarrow(1), g r-\mathcal{F C P}{ }_{n}$ is closed under direct sum and extensions. Therefore, we deduce that $\left(g r-\mathcal{F C P}{ }_{n}\right.$, $\left.g r-\mathcal{F C P}{ }_{n}^{\perp}\right)$ is a perfect hereditary cotorsion theory. If $N$ is $n$-FCP-gr-projective for any $N \in g r-\mathcal{F C P}{ }_{n}^{\perp}$, then it is clear that $N$ has an $n$-FCP-gr-projective cover with the unique mapping property.

Acknowledgment. The authors would like to thank the referee for the helpful suggestions and valuable comments.

\section{References}

[1] M. Amini, Gorenstein $\pi[T]$-projectivity with respect to a tilting module, Int. Electron. J. Algebra, 27 (2020), 114-126.

[2] M. Amini and F. Hassani, Copresented dimension of modules, Iran. J. Math. Sci. Inform., 14(2) (2019), 139-151.

[3] M. J. Asensio, J. A. Lpez Ramos and B.Torrecillas, Covers and envelopes over gr-Gorenstein rings, J. Algebra, 215 (1999), 437-459.

[4] M. J. Asensio, J. A. Lpez Ramos and B.Torrecillas, FP-gr-injective modules and gr-FC-ring, Algebra and number theory (Fez), 111, Lecture Notes in Pure and Appl. Math., 208, Dekker, New York, 2000.

[5] D. Bennis, H. Bouzraa and A. Kaed, On n-copresented modules and ncocoherent rings, Int. Electron. J. Algebra, 12 (2012), 162-174.

[6] J. Chen and N. Ding, On n-coherent rings, Comm. Algebra, 24(10) (1996), 3211-3216.

[7] D. L. Costa, Parameterizing families of non-Noetherian rings, Comm. Algebra, 22(10) (1994), 3997-4011.

[8] D. E. Dobbs, S. Kabbaj and N. Mahdou, n-coherent rings and modules, Lecture Notes in Pure and Appl. Math., 185 (1997), 269-281.

[9] J. R. García Rozas, J. A. López- Ramos and B. Torrecillas, On the existence of flat covers in R-gr, Comm. Algebra, 29(8) (2001), 3341-3349.

[10] R. Hazrat, Graded Rings and Graded Grothendieck Groups, London Mathematical Society Lecture Note Series, 2016.

[11] J. P. Jans, On co-Noetherian rings, J. London Math. Soc., (2)1 (1969), 588-590.

[12] M. Kleiner and I. Reiten, Abelian categories, almost split sequences, and comodules, Trans. Amer. Math. Soc., 357(8) (2005), 3201-3214.

[13] L. Mao, Ding-graded modules and gorenstein gr-flat modules, Glasg. Math. J., 60(2) (2018), 339-360. 
[14] C. Nastasescu, Some constructions over graded rings, J. Algebra, 120(1) (1989), 119-138.

[15] C. Nastasescu and F.Van Oystaeyen, Graded Ring Theory, North-Holland Mathematical Library 28, North-Holland Publishing Company, Amsterdam, 1982.

[16] C. Nastasescu and F.Van Oystaeyen, Methods of Graded Rings, Lecture Notes in Mathematics, 1836, Springer-Verlag, Berlin, 2004.

[17] J. Rotman, An Introduction to Homological Algebra, Second edition, Universitext, Springer, New York, 2009.

[18] X. Yang and Z. Liu, FP-gr-injective modules, Math. J. Okayama Univ., 53 (2011), 83-100.

[19] J. Zhan and Z. Tan, Finitely copresented and cogenerated dimensions, Indian J. Pure Appl. Math., 35(6) (2004), 771-781.

[20] T. Zhao, Z. Gao and Z. Huang, Relative FP-gr-injective and gr-flat modules, Internat. J. Algebra Comput., 28(6) (2018), 959-977.

[21] Z. Zhu, $n$-cocoherent rings, $n$-cosemihereditary rings and $n$-V-rings, Bull. Iranian Math. Soc., 40(4) (2014), 809-822.

[22] R. Wisbauer, Foundations of Module and Ring Theory, Revised and translated from the 1988 German edition. Algebra, Logic and Applications, 3, Gordon and Breach Science Publishers, Philadelphia, PA, 1991.

[23] W. Xue, On almost excellent extensions, Algebra Colloq., 3(2) (1996), 125-134.

[24] W. Xue, On n-presented modules and almost excellent extensions, Comm. Algebra, 27(3) (1999), 1091-1102.

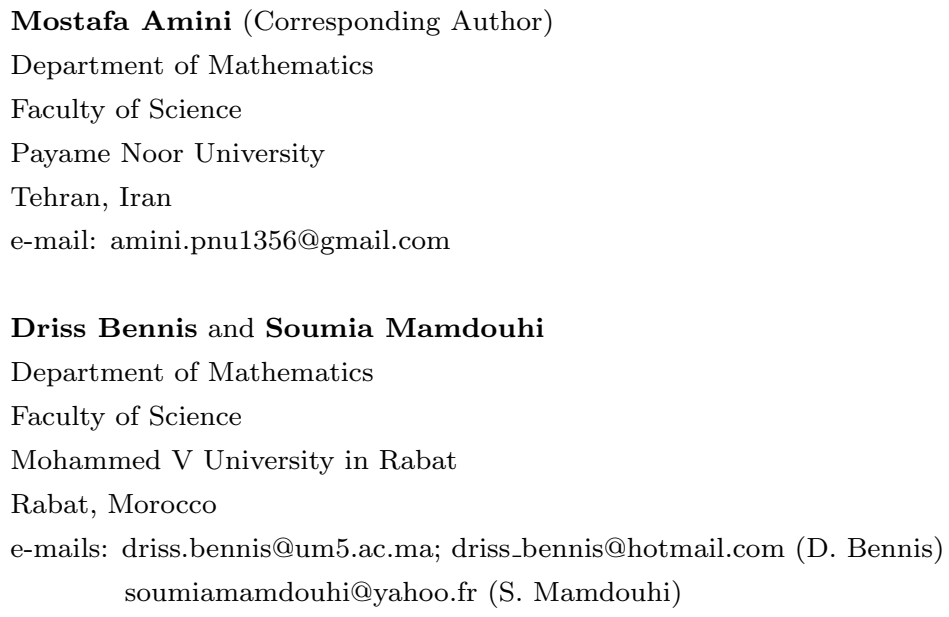

